\title{
Research on Data Processing Method of High Altitude Meteorological Parameters Based on Neural Network
}

\author{
Min Qiu ${ }^{*}, 1$, Yan Nan Mu ${ }^{1}$, Xiu Ping Zhang ${ }^{1}$ and Dong Yang ${ }^{2}$ \\ ${ }^{1}$ Heihe University, Nanjing, Jiangsu, China \\ ${ }^{2}$ Heilongjiang Institute of Technology, Heilongjiang, China
}

\begin{abstract}
The high altitude meteorological parameters include longitude, latitude, atmospheric pressure, temperature, humidity, etc., which are influenced between each parameter, therefore, it is very important to deal with and analyze these parameters. In this paper, we employ MATLAB software, research the basic algorithm of BP neural network, compare resilient BP algorithm, the Fletcher- Reeves algorithm and the proportion conjugate gradient algorithm, establish the forecast model of high altitude meteorological data with Fletcher-Reeves algorithm, and analyze the influence of the hidden layer nodes of training. Finally, we analyze the errors of the sensor models, obtain the optimal results using neural network, and ascertain the final data processing method.
\end{abstract}

Keywords: Data processing, MATLAB, meteorological parameters, neural network.

\section{INTRODUCTION}

As a big country of the World Meteorological Organization member states, it has more than 4,000 various types of meteorological stations in many departments, which accounted for $65 \%$ of the meteorological department [1]. The high-altitude meteorological observation is proceed every day, and meteorological data is very complicated, which needs to process and analyze the data to obtain more concise data, and to offer timely and accurate high-altitude meteorological data for weather forecasting, climate analysis, scientific research and international exchange.

Artificial neural network is a mathematical model algorithm, it uses a distributed parallel process information model, which is characterized by mimicking the neural network behavior of animals and the foundation is the research on brain physiology. Common models include single perception neural networks, linear neural network, BP neural network, radial basis function neural networks, selforganizing neural network and the Elman neural network etc. In this paper, we use BP neural network mainly, which is in the perception and linear neural network algorithm, used to estimate the error index of connected weights of each neuron as the difference of desired output and the actual output. In solving linear inseparable problems, meanwhile, BP neural network needs to introduce multi-level network, the major problem is how to estimate the error of each neuron in hidden layer of network. As in practical application, any desired output value of hidden layer neurons can't be known, in this case, BP neural network is proposed [2].

The main principle of BP algorithm is: output error of previous layer is estimated by the error of after layer, and then to estimate the error of more previous layer, which is an anti-pass process and will gain the complete error to obtain output error of back propagation. The error is allocated to all units, each unit with the error signal corrects the weight of the each unit in each layer, and therefore, this algorithm is called an error back propagation algorithm, is BP algorithm. The accuracy of this algorithm will be reduced with the error back propagation, but it is an effective method of neural network training algorithm, and multilayer feedforward neural networks can approximate any nonlinear function, therefore, it can be widely used in various science and technology fields [3].

\section{THE EXPERIMENT AND DATA}

The high-altitude meteorological observation observes wind direction, wind speed, air pressure, temperature, humidity and other weather elements from ground to 30,000 meters altitude. In the experiments, radiosonde is flied into air by hydrogen balloons, it induces the ambient air pressure, temperature and humidity, and transmitted back the detected GPS and meteorological data which is processed analyzed by the system terminal to the ground monitoring center by GPRS. Observers on the ground collate and calculate the received data to obtain high-altitude meteorological elements as wind direction, wind speed, air pressure, temperature and humidity. Fig. (1) is a work schematic diagram of the radiosonde $[4,5]$.

Radiosonde launches until the balloon explodes, it collected 150 sets of data, which lasted 50 minutes. Table 1 lists only 10 groups of high altitude experimental data that take once every five minutes as the data is more, which is convenient to study the law and accurate.

\section{THE ESTABLISHMENT OF HIGH-ALTITUDE METEOROLOGICAL DATA PREDICT MODEL}

\subsection{Data Preprocessing}

In order to satisfy the conditions of BP network nodes function, we should deal with the training sample that is 
Table 1. High altitude experiment data.

\begin{tabular}{|c|c|c|c|c|c|c|}
\hline Time (min) & Latitude $\left({ }^{\circ}\right)$ & Longitude $\left({ }^{\circ}\right)$ & Altitude (m) & Temperature $\left({ }^{\circ} \mathrm{C}\right)$ & Humidity (\%RH) & Pressure (kPa) \\
\hline 5 & 45.43 & 126.44 & 632 & 8.6 & 52.5 & 91.20 \\
\hline 10 & 45.43 & 126.45 & 2171 & -4.1 & 55.6 & 75.76 \\
\hline 15 & 45.42 & 126.46 & 3819 & -13.6 & 49.4 & 61.64 \\
\hline 20 & 45.40 & 126.47 & 5561 & -27.2 & 32.3 & 49.27 \\
\hline 25 & 45.38 & 126.48 & 7232 & -39.9 & 16.1 & 39.36 \\
\hline 30 & 45.36 & 126.50 & 8885 & -55.0 & 15.7 & 31.18 \\
\hline 35 & 45.33 & 126.51 & 10583 & -66.2 & 14.7 & 24.21 \\
\hline 40 & 45.31 & 126.54 & 12404 & -63.0 & 10.2 & 18.35 \\
\hline 45 & 45.29 & 126.57 & 14181 & -62.2 & 8.5 & 13.99 \\
\hline 50 & 45.28 & 127.00 & 15679 & -63.0 & 4.6 & 11.08 \\
\hline
\end{tabular}

high-altitude meteorological data we collected firstly and then make the normalization process. Processing methods include programming and invoking function directly, in invoking function, we use premnmx function, its main principle is shown in formula (1):

$W=\frac{L_{i}-v}{u-v}$

where $W$ is the normalized input data of feedforward network, $L_{i}$ is the original test data, $v$ is the minimum value of all samples sequence, $u$ is the maximum value of all samples sequence.

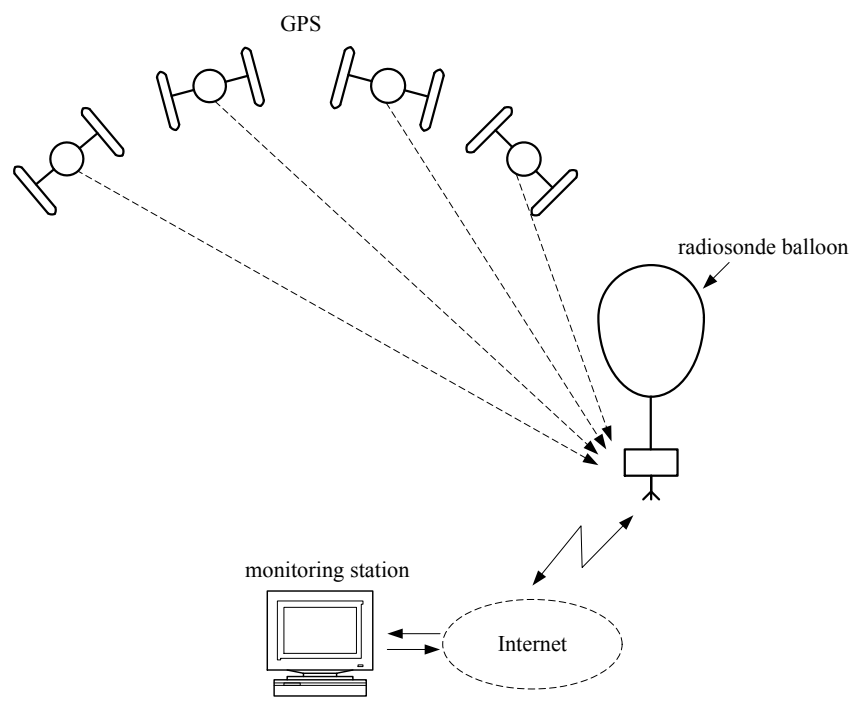

Fig. (1). Preparations of experiment.

\subsection{The Choice of Prediction Model Algorithm}

In order to make the calculation method be more practical, we select high-altitude experimental data measured by radiosonde as the analysis object. According to the time trends, we predict the next set of data through the measured exact data to overcome the phenomenon of a radiosonde data loss and ensure data integrity [6].
BP neural networks include many training functions corresponding to different algorithms. Such as batch gradient descent training function, momentum batch gradient descent function, adaptive learning algorithm, resilient BP algorithm, Fletcher-Reeves algorithm, the conjugate gradient algorithm and Quasi-Newton algorithm etc. These different algorithms call different functions. In this paper, the amount of data is not large, so we compare three algorithms with short training time and less memory, and select the final algorithm to determine the accuracy of the prediction model.

\subsubsection{Resilient BP Algorithm}

Multi-layer BP neural network transfer function is $(0,1)$ S-type. The biggest advantage of this function is the ability to map infinite input to finite output, and if the input is extremely small or extremely large, its rate will be close to zero slope. So the gradient value can be very small when we train multi-layer BP neural network with S-type, and the training efficiency of the network is improved ultimately. The purpose of resilient BP algorithm is to solve this problem, so that the gradient modulus value has no effect on the entire network learning process. The training result is shown as Fig. (2).

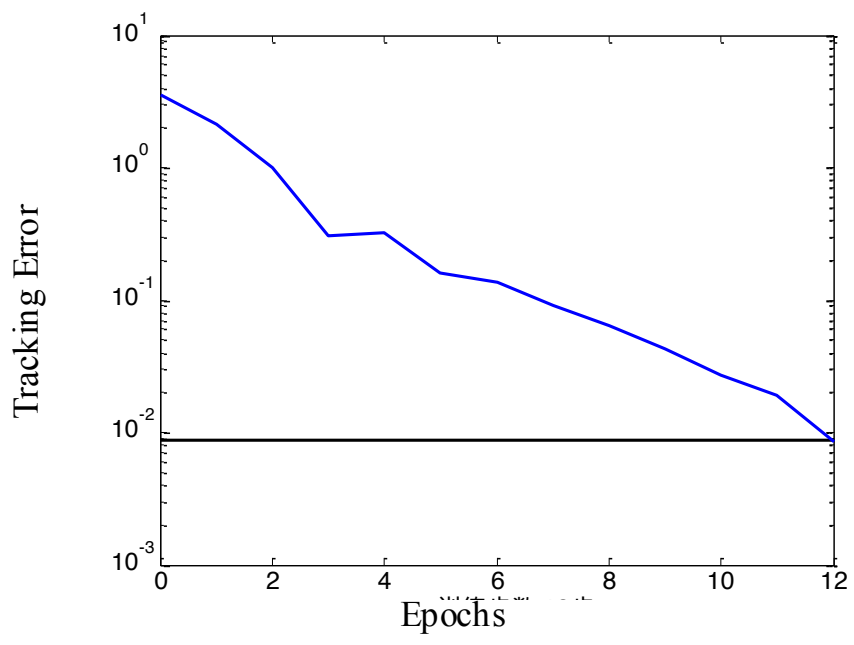

Fig. (2). Training result diagram of resilient BP algorithm. 


\subsubsection{Fletcher Reeves Algorithm}

Fletcher-Reeves algorithm is a conjugate gradient algorithm and the training function is traincgf. The training time of this algorithm is much less than adaptive modification learning algorithm, and its memory is only a little more than ordinary algorithm, therefore, in the larger weights BP neural network training, traincgf is advantageous. Fletcher-Reeves algorithm shown as formula (2) to (5):

$P_{0}=-g_{0}$

$x_{k+1}=x_{k}+\alpha_{k} P_{k}$

$P_{k}=-g_{k}+\beta_{k} P_{k-1}$

$\beta_{k}=\frac{g_{k}^{T} g_{k}}{g_{k}^{T} g_{k-1}}$

where $x_{k}$ represents the weight value of the k-th cycle, $g_{0}$ represents the gradient of the first cycle, $g_{k}$ represents the current gradient, $\alpha_{k}$ is the learning rate, $\beta_{k}$ gradient transformation parameters. The training result is shown in Fig. (3).

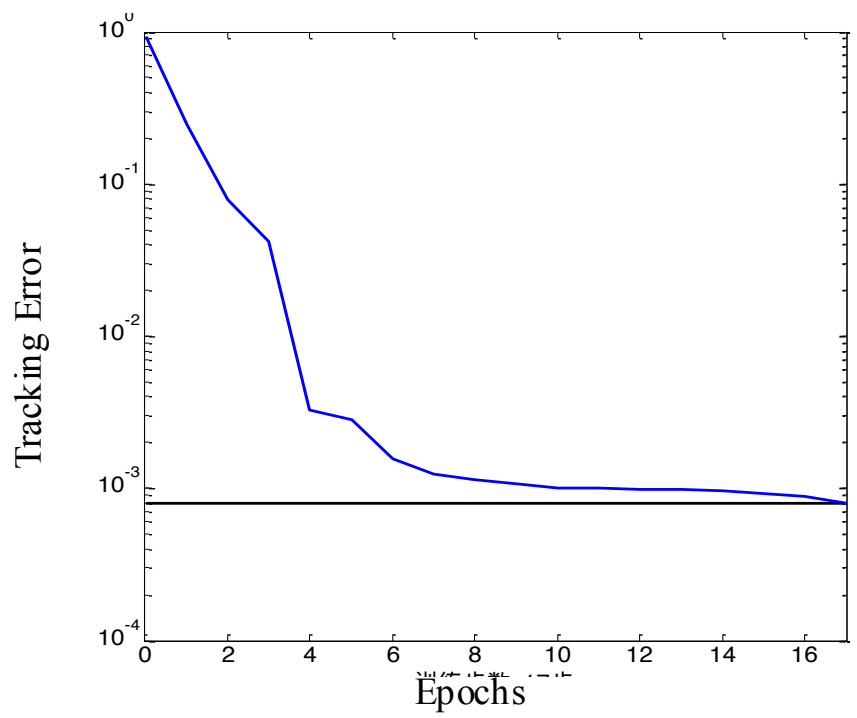

Fig. (3). Training results diagram of Fletcher-Reeves algorithm.

\subsubsection{Proportion Conjugate Gradient Algorithm}

The characteristic of conjugate gradient algorithm is that linear search for the adjust direction of the network must be done in each training cycle, it consumes a lot of time, while the proportion conjugate gradient algorithm combines modulus value trust region algorithm and conjugate gradient algorithm to reduce the search time effectively. The training function of this algorithm is trainscg. The training result is shown in Fig. (4).

\subsection{Analysis of Predict Results}

We determine the best prediction model of the three models by changing epochs, network training target error, study rate and so on. We achieve optimal training methods through changing the number of nodes in the hidden layer of the model, and determine the final prediction model. In the resilient BP algorithm, we confirm the target error and iterations by comparing the training results, the training results are shown in Fig. (5), and we obtain the error results shown in Table 2 after the simulation.

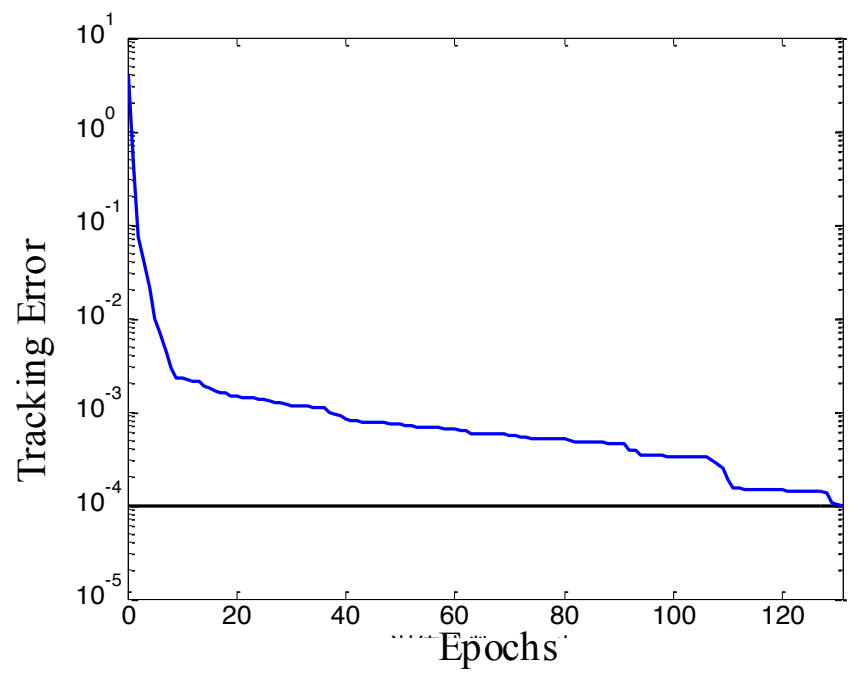

Fig. (4). Training results diagram of proportional conjugate gradient algorithm.

As Fig. (5), the effect of the training is better in 22 steps and 64 steps, and gentle convergence. From the data in the table, when the iteration is 24 , the several errors is minimized, the target error is minimized, the time of training is less, taking up less memory, simulation results are better and data can be forecasted better. Thus, we select the model which the epochs are 22 and the target error is 0.0026 to predict the accuracy of data.

Table 2. Errors results of resilient BP algorithm.

\begin{tabular}{|c|c|c|c|}
\hline Tracking Error & Epochs & MSE & SSE \\
\hline \hline 0.0028 & 10 & 0.0026 & 0.0154 \\
\hline 0.0088 & 12 & 0.0085 & 0.0511 \\
\hline 0.0026 & 22 & 0.0022 & 0.0133 \\
\hline 0.003 & 64 & 0.0028 & 0.0168 \\
\hline
\end{tabular}

In Fletcher-Reeves algorithm, we employ the same method, the training results are shown in Fig. (6), the error results are shown in Table 3.

By the results, the effect is better when the epochs are 33 and 39, smoother convergence, target error is small, and the several errors are also small. By contrast, we select the 33 epochs as the best predictive model of the algorithms.

In the proportion conjugate gradient algorithm, we also employ the same method, and the training results are shown in Fig. (7). Training results errors are shown in Table 4. The results show that four models have convergence effect and can achieve the target error requirements. By comparing, target error, standard deviation and variance are the smaller the better. We ultimately chose 310 epochs, and its effect is best. 


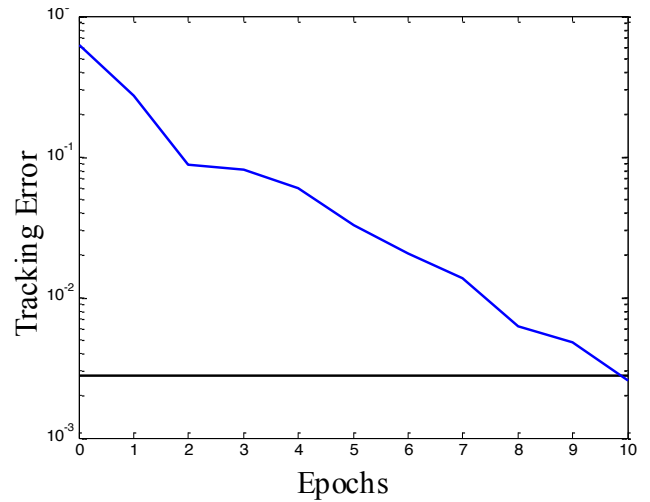

a) Training result of 10 epochs

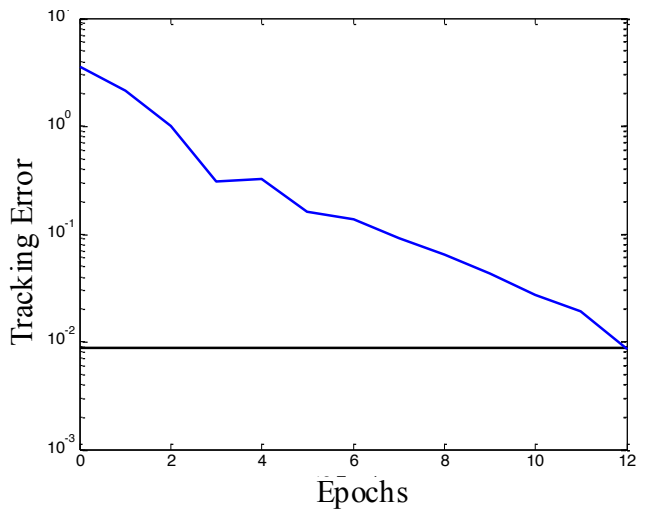

b) Training result of 12 epochs

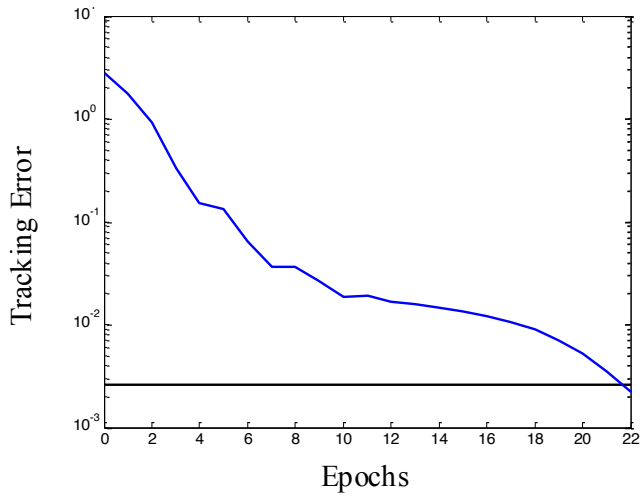

c) Training result of 22 epochs

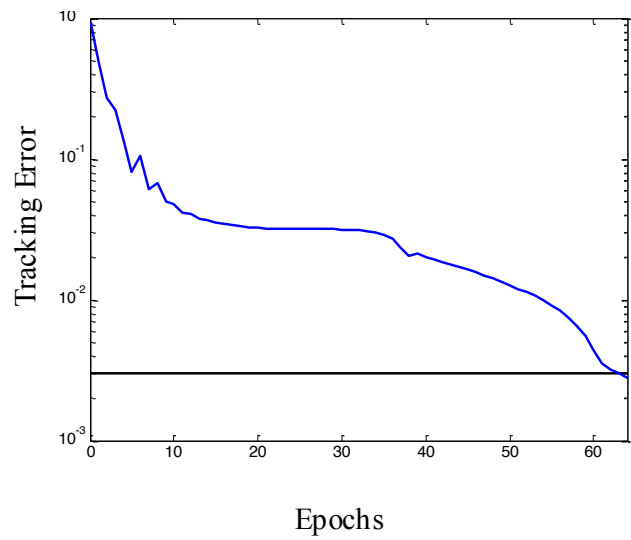

d) Training result of 64 epochs

Fig. (5). Training result diagrams of resilient BP algorithm.

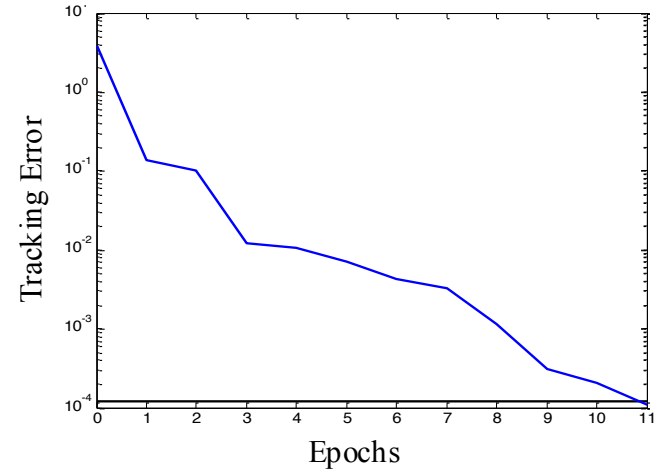

a) Training result of 11 epochs

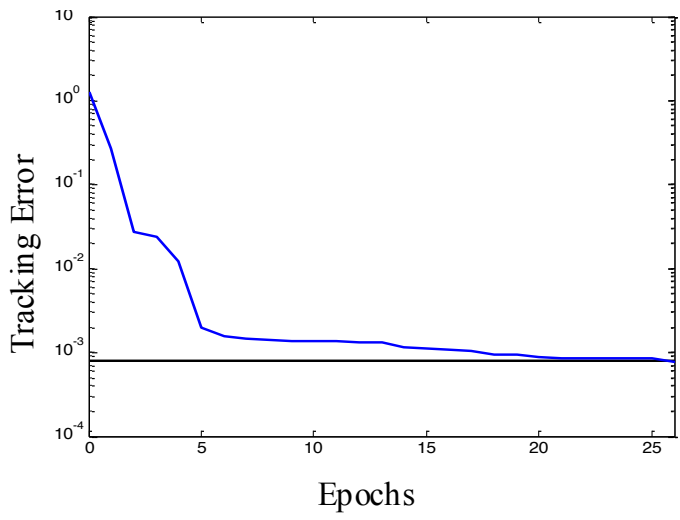

b) Training result of 26 epochs

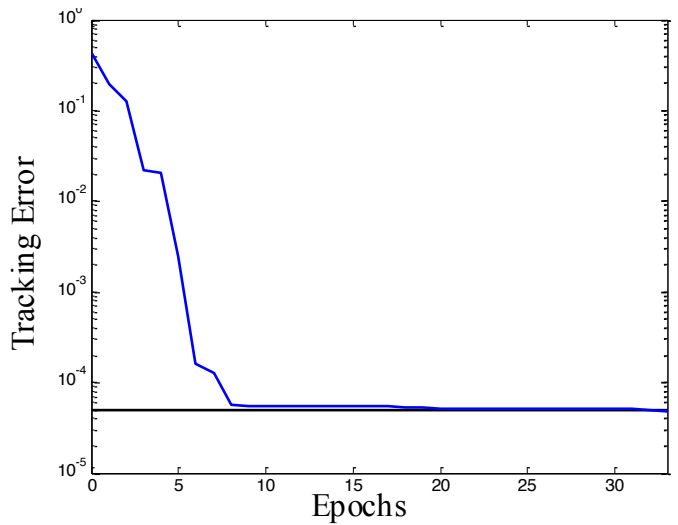

c) Training result of 33 epochs

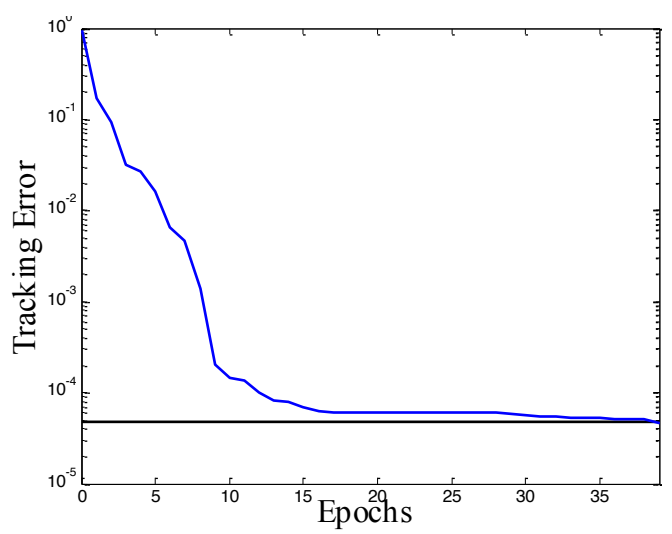

d) Training result of 39 epochs

Fig. (6). Training results diagrams of Fletcher-Reeves algorithm. 
Table 3. Errors results of Fletcher-Reeves algorithm.

\begin{tabular}{|c|c|c|c|}
\hline Tracking Error & Epochs & MSE & SSE \\
\hline \hline 0.000120 & 11 & $1.1050 \times 10^{-4}$ & $6.6279 \times 10^{-4}$ \\
\hline 0.000800 & 26 & $7.5617 \times 10^{-4}$ & $4.5000 \times 10^{-3}$ \\
\hline 0.000050 & 33 & $4.8171 \times 10^{-5}$ & $2.8902 \times 10^{-4}$ \\
\hline 0.000048 & 39 & $4.4673 \times 10^{-5}$ & $2.8037 \times 10^{-4}$ \\
\hline
\end{tabular}

Table 4. Errors results of proportional conjugate gradient algorithm.

\begin{tabular}{|c|c|c|c|}
\hline Tracking Error & Epochs & MSE & SSE \\
\hline \hline 0.00010 & 102 & $9.9316 \times 10^{-5}$ & $5.9590 \times 10^{-4}$ \\
\hline 0.00010 & 110 & $9.9826 \times 10^{-5}$ & $5.9895 \times 10^{-4}$ \\
\hline 0.00010 & 121 & $9.8264 \times 10^{-5}$ & $5.8958 \times 10^{-4}$ \\
\hline 0.00008 & 310 & $7.9849 \times 10^{-5}$ & $4.7910 \times 10^{-4}$ \\
\hline
\end{tabular}

By comparing the three algorithms, the target error of Fletcher-Reeves algorithm and the conjugate gradient algorithm proportion are small relatively, and the several errors are smaller [7]. The two algorithms are all conjugate gradient algorithms. Although the target error, mean square error and variance of the proportion conjugate gradient algorithm are smaller than the Fletcher-Reeves algorithm, its epochs are too many, computing is slower than Fletcher-
Reeves algorithm, and the error is not much smaller with respect to the order of magnitude, therefore, we decide to employ Fletcher-Reeves algorithm finally [8].

\subsection{The Influence of the Hidden Layer Nodes}

In this paper, the input nodes are six fixed factors (longitude, latitude, altitude, humidity, temperature and pressure) in the statistical prediction equation, and we compare training by changing hidden nodes. The result shows the hidden nodes of network play a large role. If the input is too small, the network can't converge or target error can't meet the requirements, while gradually increasing the hidden node will shorten the time and improve network training speed, but the forecast results are worse. If we want better forecast results and the smaller target error, we need increase the epochs. So we choose Fletcher-Reeves algorithm to train, when changing the hidden layer node, we can adjust the target error to make the training achieve convergence $[9,10]$.

From Table 5, if the best prediction is achieved, we hope the target error is small, the epochs are little, the several errors are small, then the simulation results can be closer to the expected value. When nodes increase from 1 to 4 , the target error reduces, but with the increase of epochs, the training is slowing. However from 4 to 6 , the errors reduce, the epochs also reduce. What more, through simulating, we know that when the hidden layer node is 6 , the simulation results are the same as the expected output. Fig. (8) shows the training simulation diagram of six hidden nodes; when nodes are $6,7,8$, the target errors are the same, so compared with the first nine nodes, the number of nodes is 6 , which is the best effect. Fig. (9) is the simulation result when the hidden nodes are 9.

Table 5. Training errors of different hidden node.

\begin{tabular}{|c|c|c|c|c|}
\hline Node Number of Hidden Layer & Tracking Error & Epochs & MSE & SSE \\
\hline 1 & $1.0 \times 10^{-5}$ & 19 & $8.2892 \times 10^{-6}$ & $4.9735 \times 10^{-6}$ \\
\hline 3 & $4.5 \times 10^{-9}$ & 197 & $4.1561 \times 10^{-9}$ & $2.4937 \times 10^{-8}$ \\
\hline 5 & $1.5 \times 10^{-9}$ & 263 & $1.3881 \times 10^{-9}$ & $8.3288 \times 10^{-9}$ \\
\hline 6 & $7.5 \times 10^{-10}$ & 53 & $7.2783 \times 10^{-10}$ & $4.3236 \times 10^{-9}$ \\
\hline 7 & $7.5 \times 10^{-10}$ & 165 & $7.4562 \times 10^{-10}$ & $4.4737 \times 10^{-9}$ \\
\hline 11 & $3.5 \times 10^{-11}$ & 144 & $2.3898 \times 10^{-11}$ & $1.4339 \times 10^{-10}$ \\
\hline 12 & $3.5 \times 10^{-11}$ & 128 & $3.6454 \times 10^{-11}$ & $2.1872 \times 10^{-10}$ \\
\hline
\end{tabular}




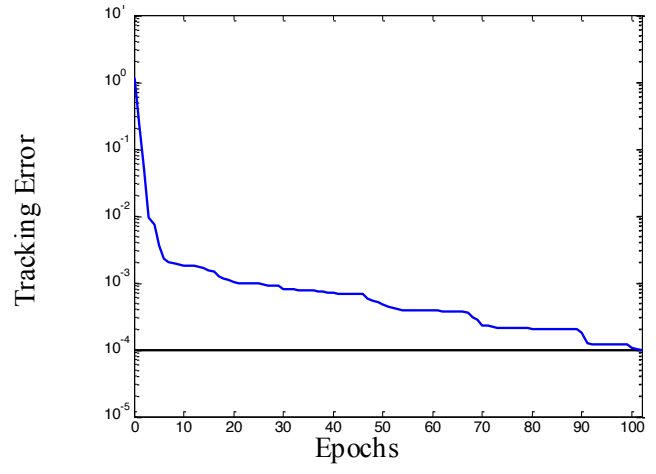

a) Training result of 102 epochs

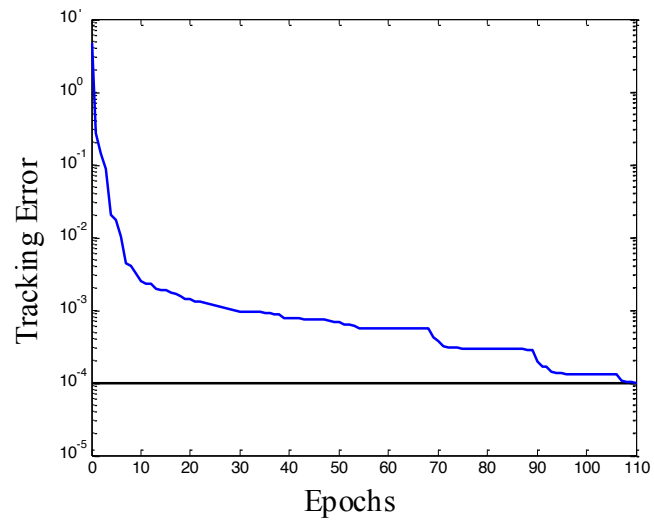

b) Training result of 110 epochs

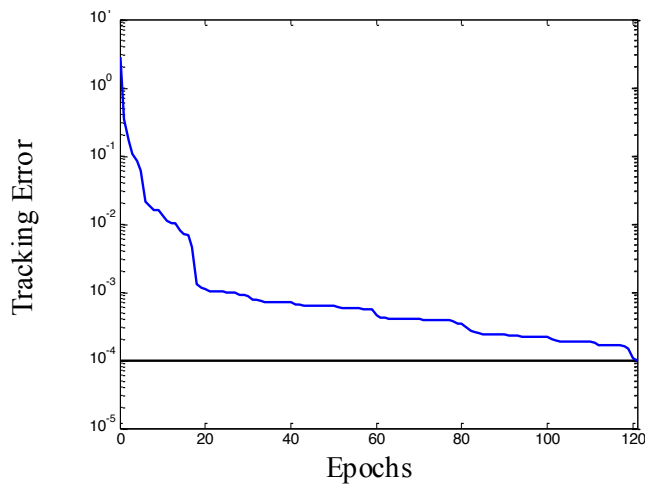

c) Training result of 121 epochs

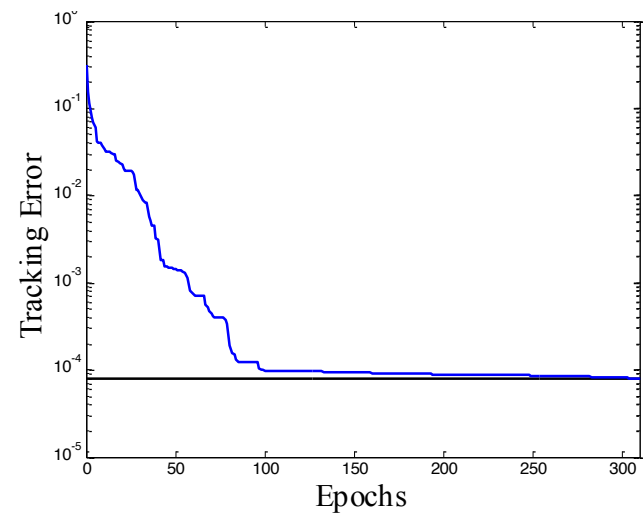

d) Training result of 310 epochs

Fig. (7). Training results diagrams of proportional conjugate gradient algorithm.

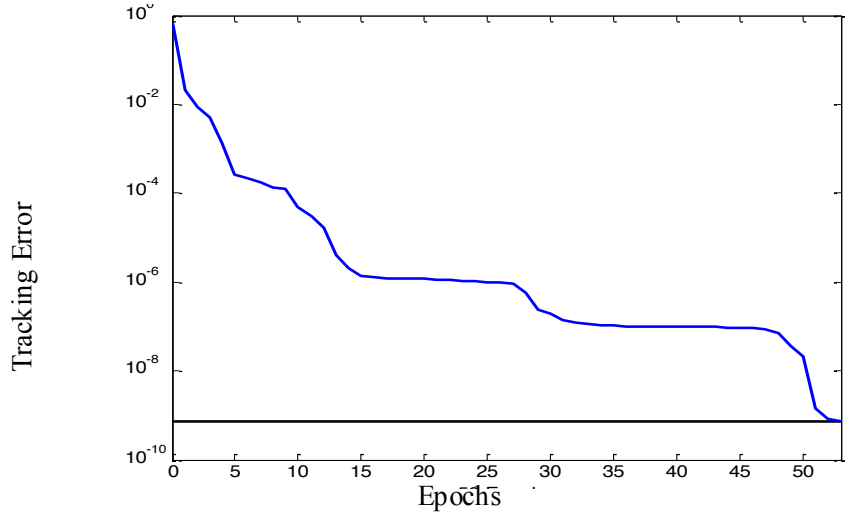

Fig. (8). Training results of 6 hidden layers neural network.

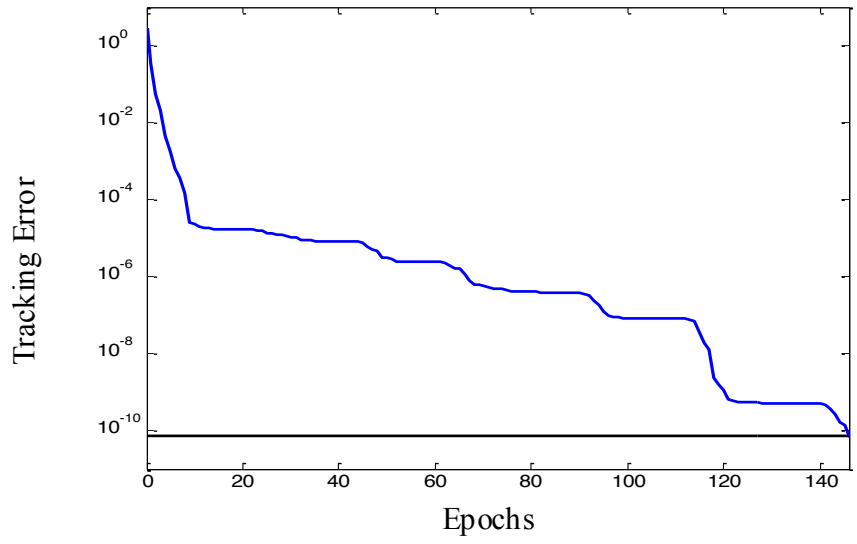

Fig. (9). Training results of 9 hidden layers neural network.

When hidden nodes are 9, the target error and several errors reduce, and the simulation results are the same as expectations; situations results of next several hidden nodes are the same as expectations. But with the increase of the nodes number, if the target error is small, we need increase epochs. So we can obtain final conclusions when hidden nodes are trained to 11 . Through analysis and training results, we ultimately select Fletcher-Reeves algorithm: we employ 6 input nodes, 6 hidden layer nodes, 1 output, 53 epochs, with the target error is $7.5 \times 10^{-10}$ to train meteorological data, and predict the missing data.

\section{SENSORS REGRESSION MODEL ERROR ANALYSIS}

Usually in the measurement, if we measure a particular object with multiple measurements, which value locate in the vicinity of the true value always. Relatively speaking from the statistical point of view, the more the number of measurements, the more the true value distribute in the vicinity. Measurements are concerned, the true value is often unknown, so we can only say that the value of the distribution more concentrated are closer to the true value. However, it is likely to the impact of the gross error, the distribution of individual position measurements deviate significantly with normal results, usually this value is called outliers. The main objective of this paper is to analyze and process data to meet the requirements of the error, to obtain more accurate weather data. 
In order to facilitate comparative analysis, in addition to directly comparative error, there are following two compared methods. Mean square and mean absolute error, as formula (6) and (7):

$$
\begin{aligned}
& M S E=\frac{1}{n} \sum_{i=1}^{n}\left(y_{i}-\hat{y}\right)^{2} \\
& M A E=\frac{1}{n} \sum_{i=1}^{n}\left|y_{i}-\hat{y}\right|
\end{aligned}
$$

Sensors in the paper include temperature, humidity, and pressure sensors. Before high-altitude meteorological observation, we need analyze the output error of each sensor, test data is shown in Table 6. Error analysis results of these three sensors are shown as Figs. (10-12).

\begin{tabular}{|c|c|c|}
\hline Test Parameter & Standard Values & Real Value \\
\hline \multirow{10}{*}{ Temperature $\left({ }^{\circ} \mathrm{C}\right)$} & -20.1 & -20.12 \\
\hline & -15.1 & -15.09 \\
\hline & -10.1 & -10.15 \\
\hline & -5.1 & -5.09 \\
\hline & -0.1 & -0.16 \\
\hline & 9.96 & 9.89 \\
\hline & 19.96 & 19.88 \\
\hline & 29.96 & 29.9 \\
\hline & 39.96 & 39.87 \\
\hline & 49.96 & 49.85 \\
\hline \multirow{5}{*}{ Humidity $(\% \mathrm{RH})$} & 9.4 & 10.8 \\
\hline & 29.7 & 30.1 \\
\hline & 49.5 & 49.9 \\
\hline & 69.4 & 70.1 \\
\hline & 88.6 & 90.2 \\
\hline \multirow{6}{*}{ Pressure $(\mathrm{kPa})$} & 2 & 2.05 \\
\hline & 20 & 19.96 \\
\hline & 40 & 39.97 \\
\hline & 60 & 59.95 \\
\hline & 80 & 79.98 \\
\hline & 100 & 99.97 \\
\hline
\end{tabular}

\section{Table 6. Test report of sensors.}

Experimental results show that the radiosonde meets the requirements of meteorological parameters within the allowable error range. Among them, the maximum deviation of temperature is $0.08^{\circ} \mathrm{C}$, maximum deviation of humidity is $0.8 \% \mathrm{RH}$, the maximum deviation of the pressure $0.08 \mathrm{kPa}$. Meet the requirements of the subject: the maximum deviation temperature does not exceed $0.1^{\circ} \mathrm{C}$, the maximum deviation of humidity does not exceed $1 \% \mathrm{RH}$, the maximum deviation of pressure does not exceed $0.1 \mathrm{kPa}$. The mean

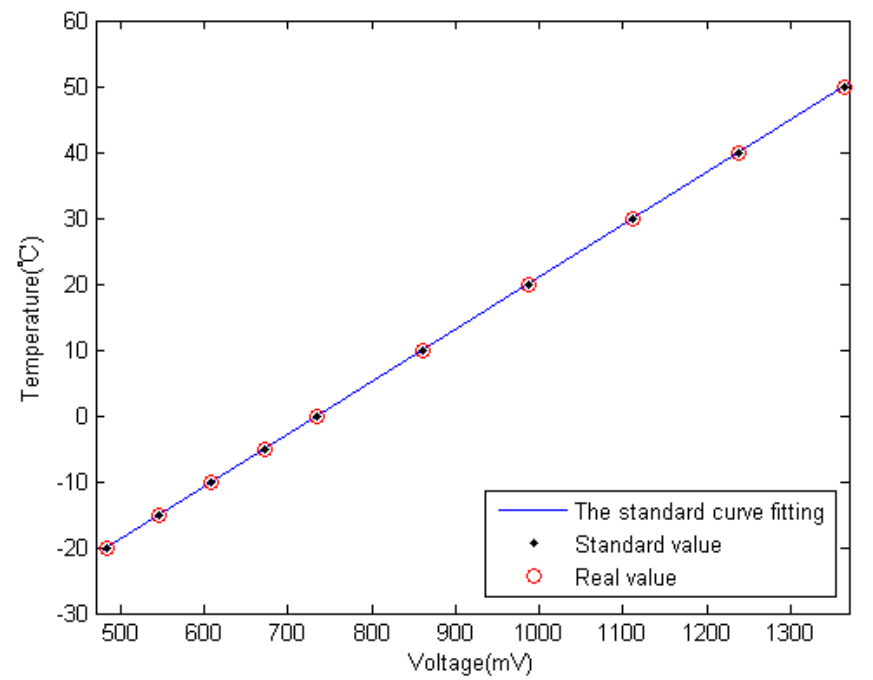

Fig. (10). Errors analysis diagram of temperature.

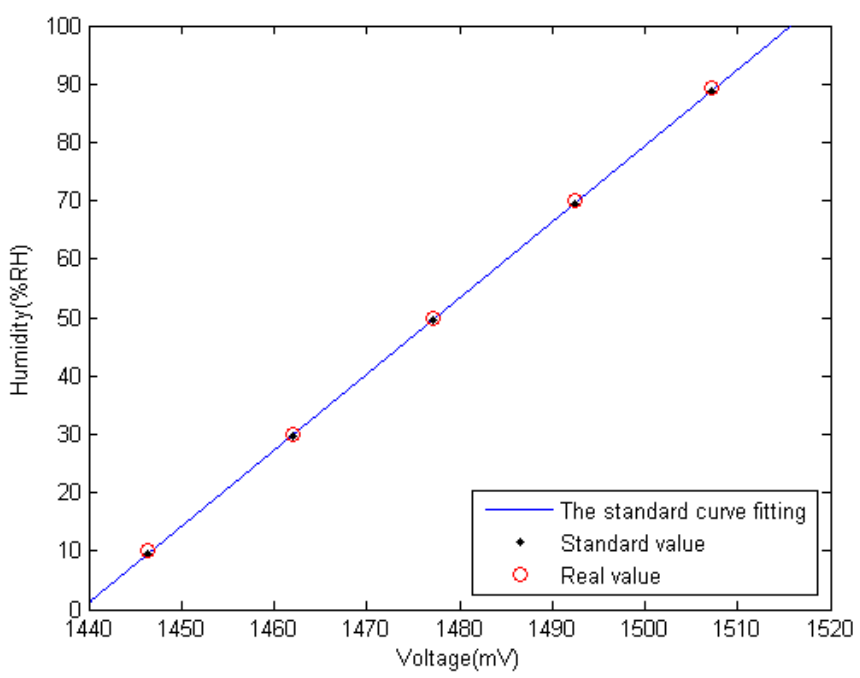

Fig. (11). Errors analysis diagram of humidity.

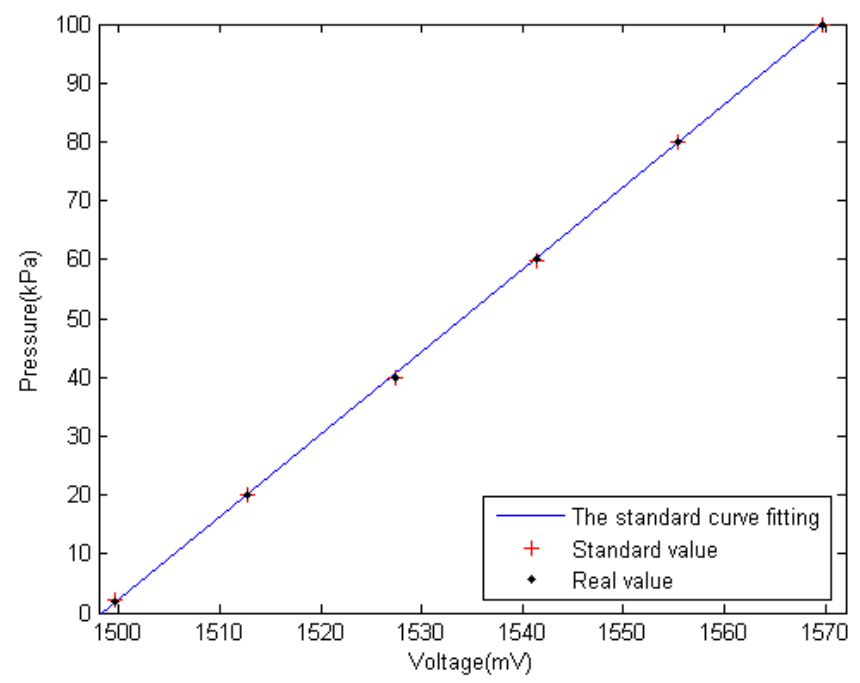

Fig. (12). Errors analysis diagram of pressure.

square, the variance and mean absolute error of each parameter are shown as Table 7 . The test data meet the requirements through error comparison. 
Table 7. Errors results table of sensors.

\begin{tabular}{|c|c|c|c|}
\hline Parameter & MSE & SSE & MAE \\
\hline \hline Temperature & 0.0042 & 0.0418 & 0.0560 \\
\hline Humidity & 0.3620 & 1.8100 & 0.5800 \\
\hline Pressure & 0.0042 & 0.0254 & 0.0633 \\
\hline
\end{tabular}

As the results of the analysis, the prediction data meet the error requirements, can achieve the training to converge, while the simulation results are the same as the expected output, indicating that the method is feasible and has a certain degree of practicality.

\section{CONCLUSION}

Meteorological data is complex and varied, in this paper, we employ MATLAB to analyze high altitude data via $\mathrm{BP}$ neural network. We study the basic algorithm BP neural network, compare and analyze resilient BP algorithm, Fletcher-Reeves algorithm and the proportion algorithm of the conjugate gradient. Then analyze the impact on training of hidden layer node, training results indicate that the effect is optimal through the predict model of high-altitude meteorological data established on Fletcher-Reeves algorithm. Meanwhile, we analyze the errors of each parameters and sensor models, the results show that neural network is very effective to process high altitude meteorological data, not only for the present study, expecting to use this method in a variety of meteorological data processing.

\section{CONFLICT OF INTEREST}

The authors confirm that this article content has no conflicts of interest.

\section{ACKNOWLEDGEMENTS}

This work is supported by the Key Project of Guangxi Social Sciences, China (No. gxsk201424), the Education
Science fund of the Education Department of Guangxi, China (No.2014JGA268), and Guangxi Office for Education Sciences Planning, China (No.2013C108).

\section{REFERENCES}

[1] Y. Qiu, Y. Shi, Y. Luo, D. Xiu, Q. Sun, C. Ge, H. Nan, "Research of high altitude meteorological data processing method," In: International Conference on Measurement, Information and Control (MIC), 2012, pp. 46-49.

[2] R. Shrivastava, M. Naja, and A. Gwal, "Study of meteorological parameters over the central Himalayan region using balloon-borne sensor," In: Proceedings of International Conference on Recent Trends in Applied Physics and Material Science: RAM 2013, 2013 , pp. 1203-1204.

[3] S. Ramachandran and M. Wang, "Near-real-time ocean color data processing using ancillary data from the Global Forecast System model," In: IEEE Transactions on Geoscience and Remote Sensing, vol. 49, pp. 1485-1495, 2011.

[4] J. Fang, J. Du, W. Xu, P. Shi, M. Li, and X. Ming, "Spatial downscaling of TRMM precipitation data based on the orographical effect and meteorological conditions in a mountainous area," Advances in Water Resources, vol. 61, pp. 42-50, 2013.

[5] Makarau, G. Palubinskas, and P. Reinartz, "Alphabet-based multisensory data fusion and classification using factor graphs," In IEEE Journal of Selected Topics in Applied Earth Observations and Remote Sensing, vol. 6, pp. 969-990, 2013.

[6] Y. Xia and J. Wang, "A general projection neural network for solving monotone variational inequalities and related optimization problems," In: IEEE Transactions on Neural Networks, vol. 15, pp. 318-328, 2004

[7] N. Merhav, "Data processing theorems and the second law of thermodynamics," In: IEEE Transactions on Information Theory, vol. 57, pp. 4926-4939, 2011.

[8] D. Warneke and O. Kao, "Exploiting dynamic resource allocation for efficient parallel data processing in the cloud," In: IEEE Transactions on Parallel and Distributed Systems, vol. 22, pp. 985997, 2011.

[9] S. Anbazhagan and N. Kumarappan, "Day-ahead deregulated electricity market price forecasting using recurrent neural network," Systems Journal, IEEE, vol. 7, pp. 866-872, 2013.

[10] J. Romo, M. Maruri, and F. Perez-Fontan, "Characterization of maximum radar reflectivity height during stratiform rain events," In: IEEE Transactions on Antennas and Propagation, vol. 60, pp. 4884-4891, 2012.

Received: September 16, 2014

Revised: December 23, 2014

Accepted: December 31, 2014

(C) Qiu et al.; Licensee Bentham Open.

This is an open access article licensed under the terms of the (https://creativecommons.org/licenses/by/4.0/legalcode), which permits unrestricted, non-commercial use, distribution and reproduction in any medium, provided the work is properly cited. 